\title{
Optical Properties of an InGaAs-InP Interdiffused Quantum Well
}

\author{
E. Herbert Li, Senior Member, IEEE
}

\begin{abstract}
A comprehensive model is developed for the calculation of polarization-dependent absorption coefficients and refractive index of the InGaAs-InP interdiffused multiple-quantumwell at room temperature for wavelengths ranging from 1.1 to $2.4 \mu \mathrm{m}$. Groups III and V types of interdiffusion are considered separately. The as-grown structure is a latticed-matched $\mathbf{I n}_{0.53} \mathbf{G a}_{0.47} \mathrm{As}-\mathrm{InP}$ structure with a well width of $60 \AA$. The optical transitions consist of a full quantum-well calculation together with $\Gamma, X$, and $L$ valleys contributions and through the Kramers-Krönig transformation to link the real and imaginary parts of the dielectric functions. The results show that GroupIII-only interdiffusion produces compressive strain and results in a band-edge red shift and refractive index enlargement, while the tensile strain induced by Group-V-only interdiffusion results in a vice verse effect. This provides a left and right tunable band edge and positive and negative index steps dependent on the interdiffusion process. A small and constant birefringence of 0.005 at around $1.55 \mu \mathrm{m}$ can also be obtained over a $50-\mathrm{nm}$ wavelength range by using Group-V-only interdiffusion. These properties have strong implications in realizing a tunable and high-performance device as well as for photonic integrations.
\end{abstract}

Index Terms - Birefringence, dielectric constants, optical refraction, quantum-well interdiffusion, quantum-well intermixing, quantum wells.

\section{INTRODUCTION}

$\mathbf{T}$ HE InGaAs-InP III-V quantum well (QW) has proven to be an important semiconductor material, particularly in the neighborhood of $1.55-\mu \mathrm{m}$ wavelengths, because of its applications in optical fiber communication. Recently, it has been demonstrated that the bandgap and perhaps the optical properties of a QW material can be engineered by thermal interdiffusion to intermix the alloy composition [1]. Two types of strain (compressive and tensile) are possible in the InGaAsP QW material system and which can be manipulated by interdiffusion. There are three possible ways of composition intermixing in the InGaAsP-InP QW: GroupIII(In, Ga atoms)-only interdiffusion [2], Group-V(As, P)only interdiffusion [3], and both group (III and V together) interdiffusion. The InGaAs-InP interdiffused QW's have been actively investigated [4]-[6], and they have also been applied to develop waveguides [7], modulators [8], and lasers [9]. This material can also be integrated monolithically with longwavelength InGaAs-InP lasers to form photonic integrated circuits [10].

The ability to modify the optical properties of these materials is important to the improved performance of device

Manuscript received May 18, 1997; revised February 17, 1998. This work was supported by the Research Grant Council earmarked grant of Hong Kong.

The author is with the Department of Electrical and Electronic Engineering, University of Hong Kong, Hong Kong.

Publisher Item Identifier S 0018-9197(98)03749-X. operations. The absorption coefficients and refractive index are two such optical parameters, the knowledge of which as a function of interdiffusion is very useful for the design of devices. The types of interdiffusion (Groups III or V) can produce a very different absorption change, refractive index step, and polarization sensitivity. Conventionally, it is rather common to use the weighted average of the refractive indexes of the well and barrier bulk materials for the refractive index of the QW multiple layers [11], [12]. In the case of absorption coefficient calculations, it is mostly done by using a bulk refractive index for the well layer material. These are inaccurate calculations and may give rise to erroneous predictions, if not misinterpretation of device performance. However, to our knowledge, there is no comprehensive model dealing with the calculation of the absolute refractive index (not change of refractive index [12], [13]) for this system. Therefore, there is a need to develop a model for the determination of the refractive index of InGaAs-InP QW's at a range of wavelengths above $1 \mu \mathrm{m}$ for both as-grown and interdiffused QW cases.

In this paper, a comprehensive model is demonstrated for the calculation of the room-temperature polarization-dependent absorption coefficients and refractive index. The model for the optical transitions employs a $\boldsymbol{k} \cdot \boldsymbol{p}$ technique to the partitioned $\Gamma, X$, and $L$ Brillouin zones of the material and through a hybrid approach. This employs a full QW calculation for the $\Gamma$ region and the application of a bulk-like calculation for the $X$ and $L$ regions, since contributions below the barrier band-edge wavelength only affect the magnitude of refractive index for wavelengths above this edge. Relevant calculations and material parameters used are weighted equally between the well and barrier throughout the model, within the band structure calculation regime, in order to make the results more reliable for device modeling. In Section II, all the theories for modeling the interdiffused QW subbands and optical parameters are shown. This is followed by Section III with results of the calculation for absorption coefficients, refractive index, birefringence, and refractive index change.

\section{MOdEl FOR THE OPTICAL PARAMETERS}

The refractive index $n$, the extinction coefficient $k$, the real and imaginary parts of the dielectric functions, $\varepsilon_{1}$ and $\varepsilon_{2}$, and the absorption coefficient $\alpha$ are the fundamental optical constants and are interrelated [14]. All the optical constants mentioned above depend on the energy band structure of the material. The relation between the band structure and $\varepsilon_{2}[15]$ is

$$
\varepsilon_{2}(E)=\frac{e^{2} \hbar^{2}}{\pi m E^{2}} \int d k^{3} M_{c v}(k)^{2} \delta\left[E_{c}(k)-E_{v}(k)-E\right]
$$


where the Dirac $\delta$ function represents the joint spectral density of states between the valence $\left[E_{v}(k)\right]$ and conduction $\left[E_{c}(k)\right]$ band states, and $M_{c v}(k)$ is the momentum matrix element between the valence and conduction band states. The real part of the dielectric function $\varepsilon_{1}$ is related to the imaginary part $\varepsilon_{2}$ by the Kramers-Krönig relations [14]

$$
\varepsilon_{1}(E)-1=\frac{2}{\pi} P V \int_{0}^{\infty} \frac{E^{\prime} \varepsilon_{2}\left(E^{\prime}\right)}{\left(E^{\prime}\right)^{2}-E^{2}} d E^{\prime}
$$

where $P V$ implies the principle part of the integral is taken. Once the knowledge of $\varepsilon_{1}$ and $\varepsilon_{2}$ is available, all the other optical constants can be calculated including the refractive index and absorption coefficients. These optical constants depend ultimately on the energy band structure of InGaAs-InP multiple quantum wells (MQW's), therefore, the model to be presented here includes contributions from the energy band structure with relevant features of the critical points.

In the following, a brief description of the model of refractive index is given. In the model, various features of the band structure at different transition energies $\left[E_{0}, E_{0}+\Delta_{0}\right.$ (correspond to $\Gamma$ valley), $E_{1}, E_{1}+\Delta_{1}$ and $E_{2}$ (correspond to $L$ and $X$ valley)] are considered. The effects of higher energy transitions which will take an important part in the calculation of the refractive index will also be discussed. In this model, interdiffusion of the group III sublattice and group V sublattice are considered separately [16]. Therefore, two types of interdiffusion profile are shown, the group III interdiffusion (only) type and the group V interdiffusion (only) type. Based on this consideration, the two interdiffusion profiles are used for the MQW [17] structure.

In the group III sublattice, the In composition profile $x(z)$ is described by

$$
\begin{aligned}
& x(z)=\frac{x_{0}}{2}\left\{2-\operatorname{erf}\left(\frac{z-c_{1}}{2 L_{d}}\right)+\operatorname{erf}\left(\frac{z-c_{2}}{2 L_{d}}\right)\right. \\
&\left.+\sum_{i=1}^{N}\left[\operatorname{erf}\left(\frac{z-a_{i}}{2 L_{d}}\right)-\operatorname{erf}\left(\frac{z-b_{i}}{2 L_{d}}\right)\right]\right\}
\end{aligned}
$$

where $x_{0}$ is the as-grown In concentration in the barrier and the cladding layer, $N$ is the number of barriers within the MQW core of the as-grown structure, $\operatorname{erf}(y)$ denotes the error function, $z \geq 0$ is the growth axis of the multiple-QW layers where the MQW structure is positioned on the positive side, $a_{i}$ and $b_{i}$ are the left and right interface positions, respectively, of the $i$ th as-grown barrier within the MQW core, while $c_{1}$ and $c_{2}$ are those at the two end positions of the as-grown MQW core (i.e., interface between the cladding and core layers).

In the group $\mathrm{V}$ sublattice, the As composition profile $y(z)$ is described by

$$
\begin{array}{r}
y(z)=1-\frac{1-y_{0}}{2}\left\{2-\operatorname{erf}\left(\frac{z-c_{1}}{2 L_{d}}\right)+\operatorname{erf}\left(\frac{z-c_{2}}{2 L_{d}}\right)\right. \\
\left.+\sum_{i=1}^{N}\left[\operatorname{erf}\left(\frac{z-a_{i}}{2 L_{d}}\right)-\operatorname{erf}\left(\frac{z-b_{i}}{2 L_{d}}\right)\right]\right\}
\end{array}
$$

where $y_{0}$ is the as-grown As concentration in the barrier and the cladding layer; other variables are defined as in (3).

\section{A. Г-Valley}

The direct edge $E_{0}$ and the spin-split levels $E_{0}+\Delta_{0}$ are considered. The contribution of the QW effect to the energy transitions is strongest in this valley. In our model, we shall first consider this in the imaginary part of the dielectric function. By considering the bound and continuum contribution from the QW material, a simple equation connecting the imaginary parts of the contributions are derived

$$
\varepsilon_{2}^{\Gamma}(\omega)=\varepsilon_{2}^{\text {exc }}(\omega)+\varepsilon_{2}^{\text {bound }}(\omega)+\varepsilon_{2}^{\Gamma \operatorname{cont}}(\omega)
$$

where $\omega$ is the angular frequency, $\varepsilon_{2}^{\Gamma}$ is the imaginary part of the dielectric function of the $\Gamma$-valley, $\varepsilon_{2}^{\text {exc }}$ is the imaginary part of the dielectric function contributed by the $1 \mathrm{~S}$ exciton effect, $\varepsilon_{2}^{\text {bound }}$ is the imaginary part of the dielectric function contributed by the conduction-valence band bound state, and $\varepsilon_{2}^{\Gamma c o n t}$ is the imaginary part of the dielectric function contributed by weighting the InP barrier and InGaAs well as a bulk material without the QW effect. The bulk material parameter is taken in a range starting at a higher energy from the band gap.

The imaginary part of the bound state dielectric function, $\varepsilon_{2}^{\text {bound }}(\omega)$ is contributed by the conduction and valence bands bound state without the electron-hole interaction. Together with the imaginary part of the dielectric function $\varepsilon_{2}^{\operatorname{exc}}(\omega)$, contributed by the exciton effect, which is derived by the density-matrix approach at the subband edge without the influence of band mixing, are given below:

$$
\begin{aligned}
\varepsilon_{2}^{\text {bound }}(\omega)= & \frac{e^{2} M_{b}^{2} \wp_{0}}{\pi \varepsilon_{0} m_{0}^{2} \omega^{2} L_{z}} \sum_{\ell, \ell^{\prime}} \int d k\left|\left\langle\psi_{C \ell} \mid \psi_{V \ell^{\prime}}\right\rangle\right|^{2} \\
& \cdot \mathcal{L}\left(E_{C \ell}\left(k_{\|}\right)-E_{V \ell^{\prime}}\left(k_{\|}\right)-\hbar \omega\right) \\
\varepsilon_{2}^{\operatorname{exc}}(\omega)= & \frac{e^{2} M_{b \wp}}{\varepsilon_{0} m_{0}^{2} \omega^{2} L_{z}}\left|\left\langle\psi_{c 1} \mid \psi_{v 1}\right\rangle\right|^{2} \\
& \times\left|\psi_{1 S}(k=0)\right|^{2} \mathcal{L}\left(E_{\mathrm{exc}}-\hbar \omega\right)
\end{aligned}
$$

where

$$
\begin{aligned}
\left\langle\psi_{c \ell} \mid \psi_{\boldsymbol{v} \ell^{\prime}}\right\rangle & =\int_{-Z_{b}}^{Z_{b}} \psi_{c \ell}(z) \psi_{\boldsymbol{v} \ell^{\prime}}(z) d z \\
\Psi_{1 S}(\rho) & =\left(4 \lambda /(2 \pi)^{1 / 2} a^{*}\right) \exp \left(-2 \rho \beta / a^{*}\right)
\end{aligned}
$$

and $E_{b}=-4 \lambda^{2} R^{*}, a^{*}=4 \pi \varepsilon \hbar^{2} /\left(\mu_{\|}^{*} e^{2}\right)$ and $R^{*}=$ $\mu_{\|}^{*} e^{4} /\left(32 \pi^{2} \varepsilon^{2} \hbar^{2}\right)$ are the exciton Bohr radius and Rydberg energy, respectively, $\varepsilon_{0}$ is the permittivity of free space, $e$ and $m_{o}$ are the electron charge and rest mass, respectively, $M_{b}^{2}=m_{o}^{2} P^{2} / 3 \hbar$ and $P$ is given by Kane's model, $k$ is the transverse wavevector in the direction parallel to the QW layer, $L_{z}$ is the as-grown QW width, $E_{p}, E_{q}$, and $E_{\text {exc }}$ are the electron subband energy, hole subband energy, and exciton transition, respectively, $\mathcal{L}$ is the Lorentzian broadening factor with HWHM $\Gamma_{b}$, and $\psi_{c}, \psi_{v}$, and $\psi_{1 s}$ are the envelope wave functions for the electron, the hole, and the exciton, respectively. the summation in (6) is over all the bound states for the conduction $(p)$ and valence $(q)$ bands; and $\wp$ is the polarization factor at the band edge.

In the calculation of $\varepsilon_{2}^{\Gamma \operatorname{cont}}(\omega)$, we have considered only the lowest direct gaps $E_{0}$ and $E_{0}+\Delta_{0}$. They are of the threedimensional (3-D) $M_{0}$-type critical points [18], [19]. Also, parabolic bands are assumed. The expression of $\varepsilon_{2}^{\Gamma \operatorname{cont}}(\omega)$ is 
given

$$
\begin{aligned}
\varepsilon_{2}^{\Gamma \operatorname{cont}}(\omega)= & {\left[\frac{2 \sqrt{\frac{2}{3}}\left(m_{\mathrm{dos}}^{*}\right)^{3 / 2} M_{b}^{2}}{(\hbar \omega)^{2}}\right] } \\
& \cdot\left[\left(\hbar \omega-E_{0}\right)^{1 / 2} H\left(\chi_{0}-1\right)\right. \\
& \left.+\frac{1}{2}\left(\hbar \omega-E_{0}-\Delta_{0}\right)^{1 / 2} H\left(\chi_{s o}-1\right)\right]
\end{aligned}
$$

where $\chi_{0}=\hbar \omega / E_{0}, \chi_{s o}=\hbar \omega /\left(E_{0}+\Delta_{0}\right), H(y)$ is the Heaviside unit step function, and $m_{\mathrm{dos}}^{*}$ is the combined density-of-states mass.

In the calculation of as-grown square $\mathrm{QW}$, the $\varepsilon_{2}^{\Gamma \operatorname{cont}}(\omega)$ is calculated by weighting its barrier and well contributions together in a ratio of well width $\left(L_{z}\right)$ to barrier width $\left(L_{b}\right)$

$$
\varepsilon_{2}^{\Gamma \operatorname{cont}}(\omega)=\frac{1}{L_{z}+L_{b}}\left[L_{z} \varepsilon_{2(\text { well })}^{\Gamma \operatorname{cont}}(\omega)+L_{b} \varepsilon_{2(\text { barrier })}^{\text {Ccont }}(\omega)\right]
$$

For the case of the interdiffused QW, each well and barrier are divided into a total of $n$ subintervals having a constant indium and arsenic concentration. In our model for the InGaAs-InP MQW structure, $n$ has been chosen as 24 , and the width of each subintervals are fixed to $0.5 \mathrm{~nm}$. $\varepsilon_{2}^{\text {Tcont }}(\omega, x, y)$ at each subintervals are calculated according to the varying In or As distribution concentration diffusion profile. The barrier layer $\varepsilon_{2}^{\text {Tont }}$ are then weighted with the well layer $\varepsilon_{2}^{\text {Tcont }}$ evenly

$$
\varepsilon_{2}^{\Gamma \operatorname{cont}}(\omega)=\frac{1}{n} \sum_{i=1}^{n}\left[\varepsilon_{2}^{\Gamma \operatorname{cont}}(\omega)\right]_{i}
$$

where $n$ is the total number of subintervals consisting of the well and barrier.

The real part of the dielectric function, $\varepsilon_{1}^{\Gamma \operatorname{cont}}(\omega)$, is determined using the Kramers-Krönig transformation of $\varepsilon_{2}^{\Gamma}(\omega)$ and is given by

$$
\begin{aligned}
\varepsilon_{1}^{\Gamma}(\omega)=1 & +\frac{1}{\pi} \int_{0}^{\infty} \frac{\varepsilon_{2}^{\Gamma}\left(\omega^{\prime}\right)}{\omega^{\prime}+\omega} d \omega^{\prime} \\
& +\frac{1}{\pi} \sum_{m=1}^{M} \int_{\omega_{m}^{\prime}}^{\omega_{m+1}^{\prime}} \frac{\varepsilon_{2}^{\Gamma}\left(\omega^{\prime}\right)}{\omega^{\prime}+\omega} d \omega^{\prime}
\end{aligned}
$$

where the second integral is a series of subdivided integrals in which the subintegration limits $\omega_{m}^{1}$ do not coincide with the $\omega$ of interest, in order to permit the evaluation of the Cauchy intergral numerically. Though the real part of the dielectric function $\varepsilon_{1}^{\Gamma}(\omega)$ is not in an analytic form, we can still calculate it through numerical means.

For the Kramers-Krönig transformation of $\varepsilon_{2}^{\Gamma}(E)$, as we cannot obtain the analytical form for $\varepsilon_{1}^{\Gamma}(E)$, only numerical integration has been performed. As integration from zero to infinity is required in the integration of the transformation, and it is impractical numerically, we employed an expression at the tail of the transition which is similar to the function of $\varepsilon_{2}^{\Gamma \operatorname{cont}}(E)$. We let the function be

$$
\Omega(E)=A_{p} E^{-1 / 2}+B_{p}
$$

\begin{tabular}{|c|c|}
\hline & $\operatorname{In}_{x} \mathrm{Ga}_{1-x} \mathrm{As} / \mathrm{InP}_{1-\mathrm{y}}$ \\
\hline $\mathrm{E}_{g}(\mathrm{x})(\mathrm{cV})$ & $\begin{array}{c}1.35-1.17 y+0.668(1-x)-0.069 y(1-x)+0.18 y^{2}+0.03(1- \\
x) y^{2}+0.758(1-x)^{2}-0.322 y(1-x)^{2}\end{array}$ \\
\hline$\Delta_{0}(x)(e V)$ & $0.34(1-x) y+0.41 x y+0.08(1-x)(1-y)+0.11 x(1-y)$ \\
\hline $\mathrm{E}_{1}(\mathrm{x})(\mathrm{eV})$ & $3.473(1-x) y+1.573 x y+3.174(1-x)(1-y)+0.3548 x(1-y)$ \\
\hline$\Delta_{1}(x)(e V)$ & $-0.5667(1-x) y+1.187 x y+0.1262(1-x)(1-y)+2.207 x(1-y)$ \\
\hline$E_{2}(x)(e V)$ & $4.525(1-x) y+4.306 x y+4.736(1-x)(1-y)+4.382 x(1-y)$ \\
\hline $\mathrm{E}_{\mathrm{g}}^{\mathrm{in}}(\mathrm{x})(\mathrm{eV})$ & $1.517(1-x) y+0.9695 x y+2.045(1-x)(1-y)+1.159 x(1-y)$ \\
\hline $\mathrm{A}\left(\mathrm{eV}^{3 / 2}\right)$ & $0.4774(1-x) y+2.013 x y+5.382(1-x)(1-y)+7.109 x(1-y)$ \\
\hline $\mathrm{B}_{1}$ & $27.89(1-x) y-21.98 x y+5.802(1-x)(1-y)-59.83 x(1-y)$ \\
\hline $\mathrm{B}_{2}$ & $-23.27(1-x) y+28.77 x y-0.5643(1-x)(1-y)+61.28 x(1-y)$ \\
\hline $\mathrm{C}$ & $4.343(1-x) y+1.761 x y+1.078(1-x)(1-y)+3.315 x(1-y)$ \\
\hline D & $376.2(1-x) y-368.1 x y+56.91(1-x)(1-y)-839.1 x(1-y)$ \\
\hline$\varepsilon_{\infty}$ & 2.1 \\
\hline & $0.1139(1-x) y+0.4061 x y+0.0817(1-x)(1-y)+0.6544 x(1-y)$ \\
\hline$m_{e}^{*}(x) / m_{0}$ & $0.0632(1-x) y+0.0213 x y+0.17(1-x)(1-y)+0.077 x(1-y)$ \\
\hline$m_{\perp \perp H}(x) / m_{0}$ & $0.088(1-x) y+0.024 x y+0.16(1-x)(1-y)+0.12 x(1-y)$ \\
\hline $\mathrm{m}_{\perp \mathrm{HI}}(\mathrm{x}) / \mathrm{m}_{0}$ & $0.5(1-x) y+0.41 x y+0.54(1-x)(1-y)+0.6 x(1-y)$ \\
\hline $\mathrm{m}_{\mathrm{L} . \mathrm{H}}^{*}(\mathrm{x}) / \mathrm{m}_{0}$ & $0.23(1-x) y+0.082 x y+0.34(1-x)(1-y)+0.29 x(1-y)$ \\
\hline $\mathrm{m}$ & $0.11(1-x) y+0.031 x y+0.19(1-x)(1-y)+0.15 x(1-y)$ \\
\hline$\varepsilon(\mathrm{x}) / \varepsilon_{0}$ & $13.18(1-x) y+14.55 x y+11.1(1-x)(1-y)+12.35 x(1-y)$ \\
\hline
\end{tabular}

where $A_{p}$ and $B_{p}$ are constants to be determined. We have only calculated $\varepsilon_{2}^{\Gamma}(E)$ from $E=0.5$ to $30 \mathrm{eV}$. The obtained
TABLE I

Material Parameters Used For InGaAs-InP QW, $m_{0}$ AND $\varepsilon_{0}$ ARE THE Electron Mass and Static Dielectric Constant, Respectively, in Free Space

TABLE II

FitTing Parameter FoR $\mathrm{IN}_{0.53} \mathrm{GA}_{0.47}$ As-INP GROUP III INTERDIFFUSED QW $\varepsilon_{2}^{\Gamma \text { cont }}$ (ROOM TEMPERATURE)

\begin{tabular}{c|c|c}
\hline $\operatorname{Ld}(\dot{\mathrm{A}})$ & $A_{p}$ & $B_{p}$ \\
\hline 0 & 0.56927 & -0.072514 \\
10 & 0.56238 & -0.071136 \\
20 & 0.55664 & -0.069988 \\
30 & 0.54804 & -0.068268 \\
\hline
\end{tabular}

$\varepsilon_{1}^{\Gamma}(E)$ is thus smaller than the theoretical result. We use the value of $\varepsilon_{2}^{\Gamma}(E)$ at 25 and $30 \mathrm{eV}$ to determine $A_{p}\left(\mathrm{eV}^{1 / 2}\right)$ and $B_{p}$, the constants of $Z(E)$

$$
\begin{aligned}
& \Omega(E=25 \mathrm{eV})=A_{p}\left(25^{-1 / 2}\right)+B_{p}=\varepsilon_{2} \Gamma(25) \\
& \Omega(E=30 \mathrm{eV})=A_{p}\left(30^{-1 / 2}\right)+B_{p}=\varepsilon_{2} \Gamma(30) .
\end{aligned}
$$

By solving (13) and (14), $A_{p}$ and $B_{p}$ can then be obtained. The tail part of $\varepsilon_{2}^{\Gamma}(E)$ is then substituted by $\Omega(E)$. The Kramers-Krönig transformation can now be readily performed:

$$
\varepsilon_{1}^{*}(E)_{(\text {tail })}-1=\frac{2}{p} P V \int_{30}^{\infty} \frac{E^{\prime} \Omega\left(E^{\prime}\right)}{\left(E^{\prime}\right)^{2}-E^{2}} d E^{\prime}
$$

where $P V$ implies the principle part of the integral is taken. A relation is then obtained

$$
\varepsilon_{1}^{\Gamma}(E)=\varepsilon_{1}^{\Gamma}(E \leq 30 \mathrm{eV})_{(\text {edge })}+\varepsilon_{1}^{*}(E)_{(\text {tail })} .
$$

By using the function $\Omega(E)$ to model the tail part of the $\varepsilon_{2}^{\Gamma}$ spectrum, the entire spectrum is used for performing the Kramers-Krönig transformation. Therefore, $\varepsilon_{1}^{\Gamma}$ can be calculated accurately. We have worked out the values of $A_{p}$ and $B_{p}$ for the MQW case of $L_{z}=60 \AA$ and $L_{d}$ (group III or $\mathrm{V})=0,10,20$, and $30 \AA$ for references. All the general material parameters for the InGaAsP are listed in Table I. Tail spectra parameters $\left(A_{p}\right.$ and $\left.B_{p}\right)$ for group III and $\mathrm{V}$ interdiffusion are listed in Tables II and III, respectively. 
TABLE III

Fitting Parameter for $\mathrm{IN}_{0.53} \mathrm{GA}_{0.47}$ As-InP GrouP $\mathrm{V}$ INTERDIFFusEd QW $\varepsilon_{2}^{\Gamma \text { cont }}$ (ROOM TEMPERATURE)

\begin{tabular}{c|c|c}
\hline $\operatorname{Ld}(\AA)$ & $A_{p}$ & $B_{p}$ \\
\hline 0 & 0.56927 & -0.072514 \\
10 & 0.596242 & -0.077908 \\
20 & 0.65856 & -0.090372 \\
30 & 0.69667 & -0.097994 \\
\hline
\end{tabular}

\section{B. L Regions}

We assume that the QW effect can be neglected in this regions. Band structure calculations indicated that the $E_{1}$ and $E_{1}+\Delta_{1}$ transitions take place along the $\langle 111\rangle$ directions or at $L$ points in the Brillouin zone. These critical points are of the $M_{1}$ type. The longitudinal effective mass, nevertheless, is much larger than its transverse counterparts and one can treat these critical points as two-dimensional (2-D) minima. Expressions of the real and imaginary parts of the dielectric function contributed by $L$ valley are given by

$$
\begin{aligned}
& \varepsilon_{1}^{L}(\omega)=-B_{1} \chi_{1}^{-2} \ln \left(1-\chi_{1}^{2}\right)-B_{2} \chi_{1 s}^{-2} \ln \left(1-\chi_{1 s}^{2}\right) \\
& \varepsilon_{2}^{L}(\omega)=\pi\left[B_{1} \chi_{1}^{-2} H\left(\chi_{1}-1\right)-B_{2} \chi_{1 s}^{-2} H\left(\chi_{1 s}-1\right)\right]
\end{aligned}
$$

where $\chi_{1}=\hbar \omega / E_{1}, \chi_{1 s}=\hbar \omega /\left(E_{1}+\Delta_{1}\right)$, and $B_{1}, B_{2}$ are the strength parameters. The weighting method used in the $\Gamma$ valley is applied to (17) and (18).

\section{X Regions}

The transition in this region is usually labeled as $E_{2}$. The nature of the $E_{2}$ transitions is more complicated, since it does not correspond to a single well-defined critical point. It has been suggested this is due to the transitions at the $\Gamma$ point in the Brillouin zone or to transitions at the $X$ point or perhaps both. More likely, there are several transitions involving both $M_{1}$ and $M_{2}$ type critical points which combine to form a peak in the joint density of states. We model the contributions of the feature labeled $E_{2}$ as a damped harmonic oscillator. The expression is given as

$$
\begin{aligned}
& \varepsilon_{1}^{X}(\omega)=C\left(1-\chi_{2}^{2}\right)^{2} /\left[\left(1-\chi_{2}^{2}\right)^{2}+\chi_{2}^{2} \gamma^{2}\right) \\
& \varepsilon_{2}^{X}(\omega)=C \chi_{2} \gamma /\left[\left(1-\chi_{2}^{2}\right)^{2}+\chi_{2}^{2} \gamma^{2}\right)
\end{aligned}
$$

where $\chi_{2}=\hbar \omega / E_{2}, C$ is the strength parameters, $\gamma$ is the damping factor, and $E_{2}$ is the transition energy. The weighting method mentioned in the $\Gamma$ valley part is also applied in the $X$ regions for (19) and (20).

\section{Indirect Gap Transitions}

The contribution from an indirect gap transition $E_{g}^{I D}$ is taken into account. Using a perturbation calculation and consideration of higher energy cutoff $E_{c}$, the expression for the contribution is

$$
\varepsilon_{2}^{I D}(\omega)=\frac{D}{(\hbar \omega)^{2}}\left(\hbar \omega-E_{g}^{I D}+\hbar \omega_{q}\right)^{2} H\left(1-\chi_{g}\right) H\left(1-\chi_{c}\right)
$$

where $\chi_{g}=\left(E_{g}^{I D}-\hbar \omega_{q}\right) / \hbar \omega, \chi_{c}=\hbar \omega / E_{c}$, and $\hbar \omega_{q}$ is the phonon energy. The cutoff energy is set to $E_{1}$.

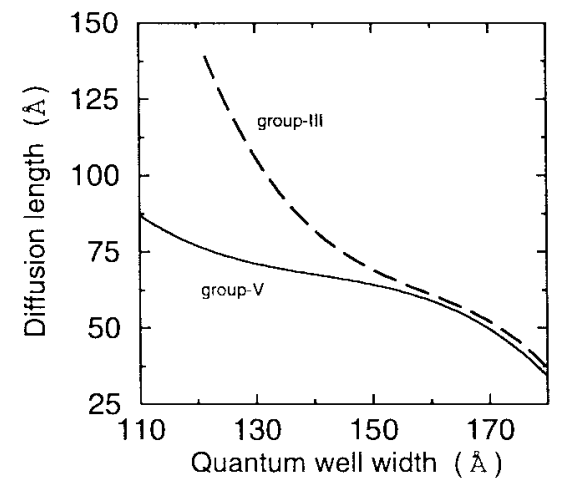

Fig. 1. The critical diffusion length (forming dislocations) against the as-grown well width for Group-III and Group-V interdiffusion.

\section{E. Other Transitions}

Higher energy transitions also make contributions to the dielectric function. However, the contributions should vary weakly with photon energy. Therefore, we model the effects of all of these transitions as a single real additive constant $\varepsilon_{\infty}$ which is not the true high-frequency dielectric constant of InGaAs-InP but simulates the effects of the higher energy transitions.

All the real parts of the dielectric functions from $\Gamma, L$, and $X$ regions and higher energy transitions are summed to obtain the total dielectric function

$$
\begin{aligned}
& \varepsilon_{1}(\omega)=\varepsilon_{1}^{\Gamma}(\omega)+\varepsilon_{1}^{L}(\omega)+\varepsilon_{1}^{X}(\omega)+\varepsilon_{\infty} \\
& \varepsilon_{2}(\omega)=\varepsilon_{2}^{\Gamma}(\omega)+\varepsilon_{2}^{L}(\omega)+\varepsilon_{2}^{X}(\omega)+\varepsilon_{2}^{I D} .
\end{aligned}
$$

The real part of $n_{r}$ is now given by

$$
n(\omega)=\left(\frac{1}{2} \varepsilon_{1}(\omega)+\frac{1}{2}\left\{\left[\varepsilon_{1}(\omega)\right]^{2}+\left[\varepsilon_{2}(\omega)\right]^{2}\right\}^{1 / 2}\right)^{1 / 2} .
$$

\section{RESUlTS AND DisCUSSION}

The theoretical results of the TE and TM polarized optical parameters (absorption coefficients and refractive index) at room temperature for interdiffused $\mathrm{QW}$ with $L_{d}$ values equal $0,10,20$, and $30 \AA$ in the $1.55-\mu \mathrm{m}$ wavelength range of interest are analyzed. The as-grown $\mathrm{QW}$ structure consists of $\mathrm{In}_{0.53} \mathrm{Ga}_{0.47} \mathrm{As}$ well layers and InP barrier layers with width $L_{z}=60 \AA$ A. Both Groups III and V interdiffusions are considered separately first, and then they will be considered together with a relative ratio. Once the as-grown lattice-matched QW structure is being interdiffused, strain will be introduced and therefore the critical layer thickness requirement for realizing a dislocation-free pseudomorphic QW material is essential. The interdiffusion-induced strain can be modeled in a simple manner by using the lattice misfit parameter. The critical layer thickness for pseudomorphic epitaxy is derived by considering the thickness dependence of the strain energy and dislocation energy and by minimizing the total energy [20]. The allowed critical layer thickness for diffusion length as a function of as-grown well thickness is shown in Fig. 1. This allowable critical-diffusion length is larger for smaller as-grown widths and more enhanced for the Group-III interdiffusion case. The extent of intermixing for maintaining a good quality material provides a more than half of the diffusion length to the 


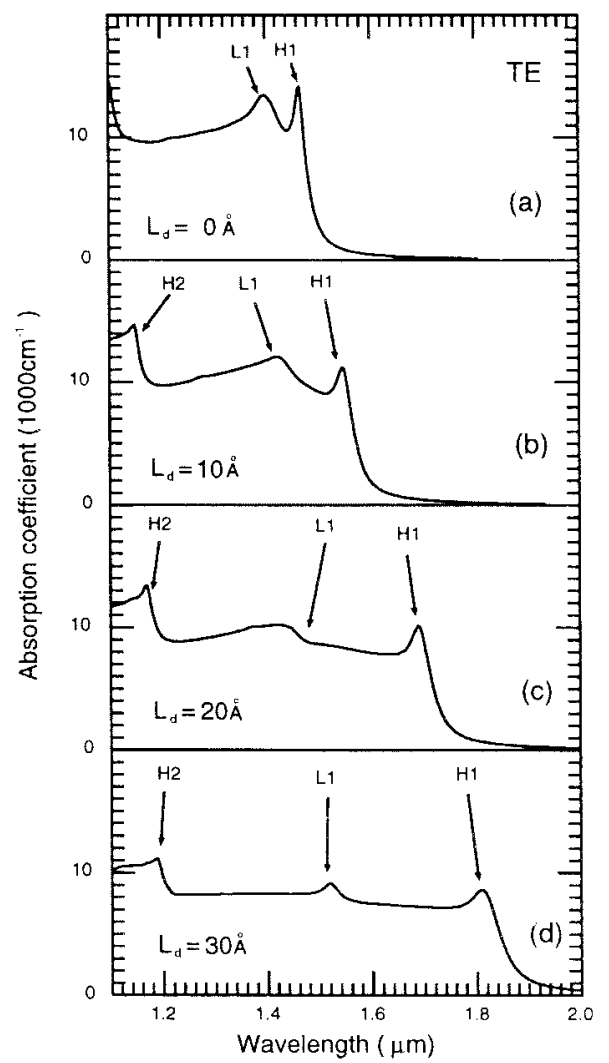

Fig. 2. TE polarization absolute refractive index spectra for several Group-III interdiffusion lengths. $L_{d}=$ (a) 0 , (b) $10 \AA$, (c) $20 \AA$, and (d) $30 \AA$.

as-grown width, and even more so in the case of GroupIII interdiffusion. This modification (tuning) of the optical parameter should provide a wide range of wavelength interest for most practical purposes.

The Group-III interdiffused TE and TM polarized absorption coefficients are shown in Figs. 2 and 3, respectively, for various cases of $L_{d}$. As can be seen, in the case of TE, the HH1 exciton transition remains strong throughout the interdiffusion and which resolves up to $L_{d}=30 \AA$ where the other bound states reveal their identity clearly. The distinct feature is a red shift of the heavy (H1) and light (L1) holes exciton states. Both of these are a consequence of the Group III interdiffusion, which produces a compressive strain [21], [22] in the well. The compressive strain is known to enhance the heavy and light hole separation as well as a reduction of the bandgap [16]. In the case of TM (see Fig. 3), since there is only one light-hole bound state, only one exciton peak can be obtained; the heavy hole is not sensitive to this polarization. The L1 band edge also undergoes a red shift with interdiffusion.

The polarized absorption coefficients subject to Group V interdiffusion are shown in Figs. 4 (TE) and 5 (TM). The effect of this interdiffusion produces a tensile strain in the well [23] and thus enlarges the band-gap [16]. A more distinct feature can be seen, as interdiffusion proceeds, where the L1 and H1 states first merged [see Fig. 4(a)], and then cross over [see Fig. 4(c)] to become a light-hole-like band edge. The L1 state eventually merges with the $\mathrm{H} 1$ state when interdiffusion gets extensive. In the case of TM (see Fig. 5), the band-edge

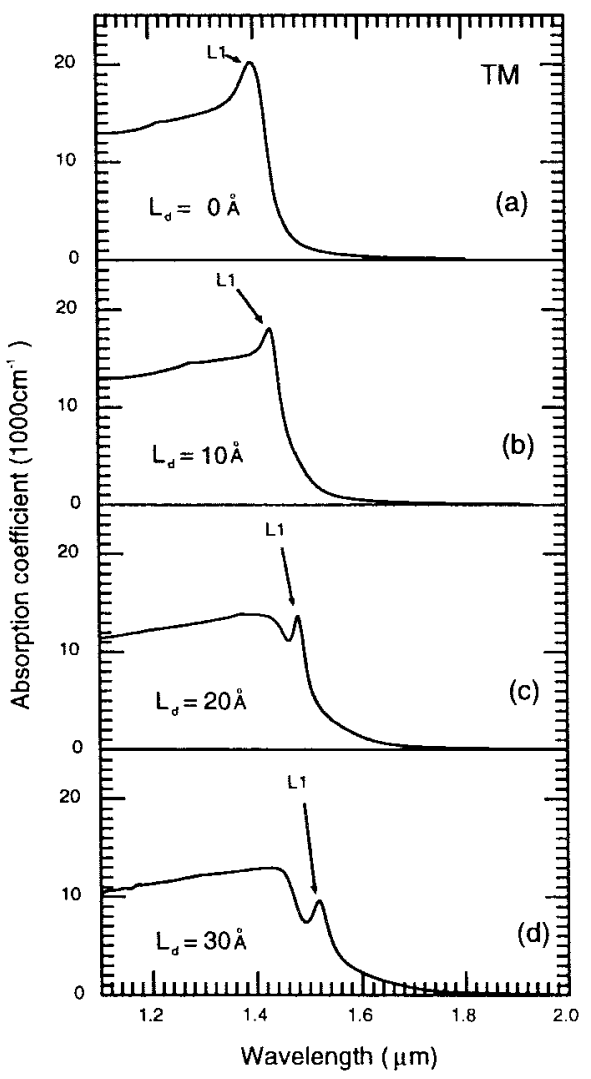

Fig. 3. TM polarization absolute refractive index spectra for several Group-III interdiffusion lengths. $L_{d}=$ (a) 0 , (b) $10 \AA$, (c) $20 \AA$, and (d) $30 \AA$.

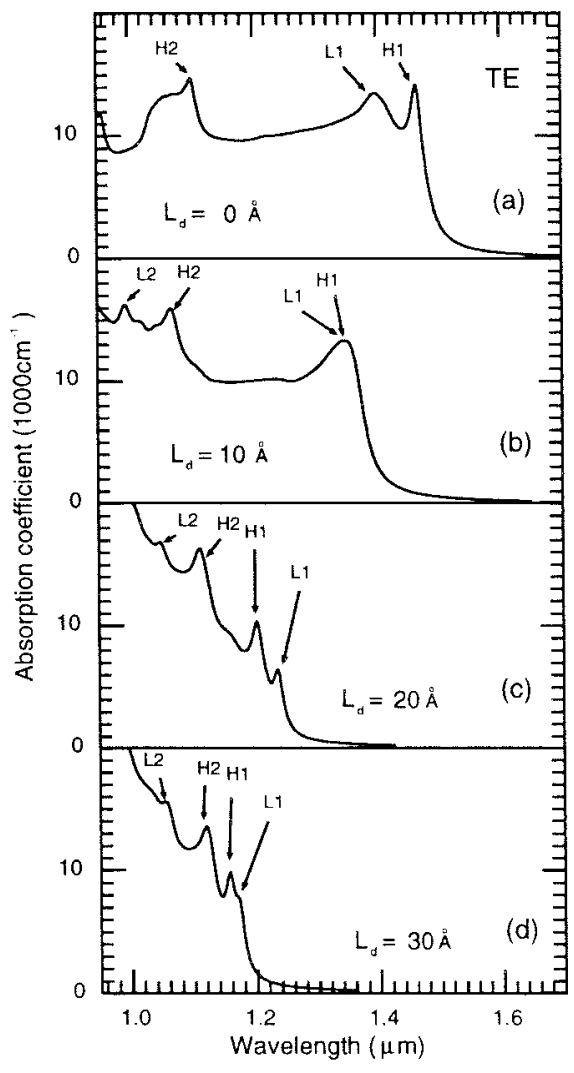

Fig. 4. TE polarization absolute refractive index spectra for several Group V interdiffusion lengths. $L_{d}=$ (a) 0, (b) $10 \AA$, (c) $20 \AA$, and (d) $30 \AA$. 


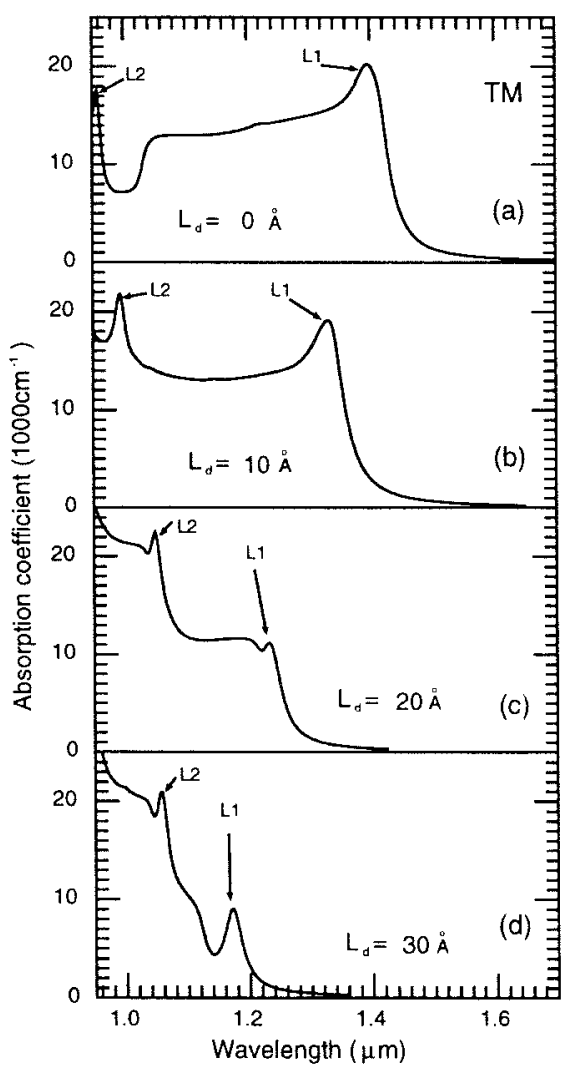

Fig. 5. TM polarization absolute refractive index spectra for several Group V interdiffusion lengths. $L_{d}=$ (a) 0 , (b) $10 \AA$, (c) $20 \AA$, and (d) $30 \AA$.

L1 exciton blue shifts and remains resolved at $L_{d}=30 \AA$, although at a much reduced magnitude.

In general, the compressive strain produced by the Group III interdiffusion pulls apart the excitonic transitions while the tensile strain produced by Group V interdiffusion pushes the transitions together as revealed in the absorption spectra. The theoretical calculation determined here is in good agreement with the measured photoluminescence [23] and photocurrent spectra [24].

The refractive index spectra for the TE polarization (Group III interdiffusion) in the wavelength range $1.4-2.4 \mu \mathrm{m}$ is shown in Fig. 6(a). The refractive index spectra for the different cases of diffusion lengths rise with decreasing wavelength from the long-wavelength side. The spectra first reach a peak due to the exciton edge at the fundamental bandgap $E_{0}$. This primary peak signifies a similar dispersion which corresponds to the $E_{0}$ edge of the bulk $\Gamma$ valley. The fine structure between the primary peak and barrier edge is due to the contributions from different bound state transitions in the QW. As diffusion length increases, the spectra and the exciton peak shifting to the longer wavelength side (red shift) are observed. The reason is similar to those observed in the absorption spectra. The TM spectra for Group III interdiffusion are shown in the same wavelength range. As in the case of TE polarization, the refractive index spectra for increasing diffusion length red shift toward longer wavelengths. However, the TM exciton peak wavelength shift is much less than the TE spectra. This is because the light hole exciton transition energy varies less sensitively with interdiffusion due to a much shallower well
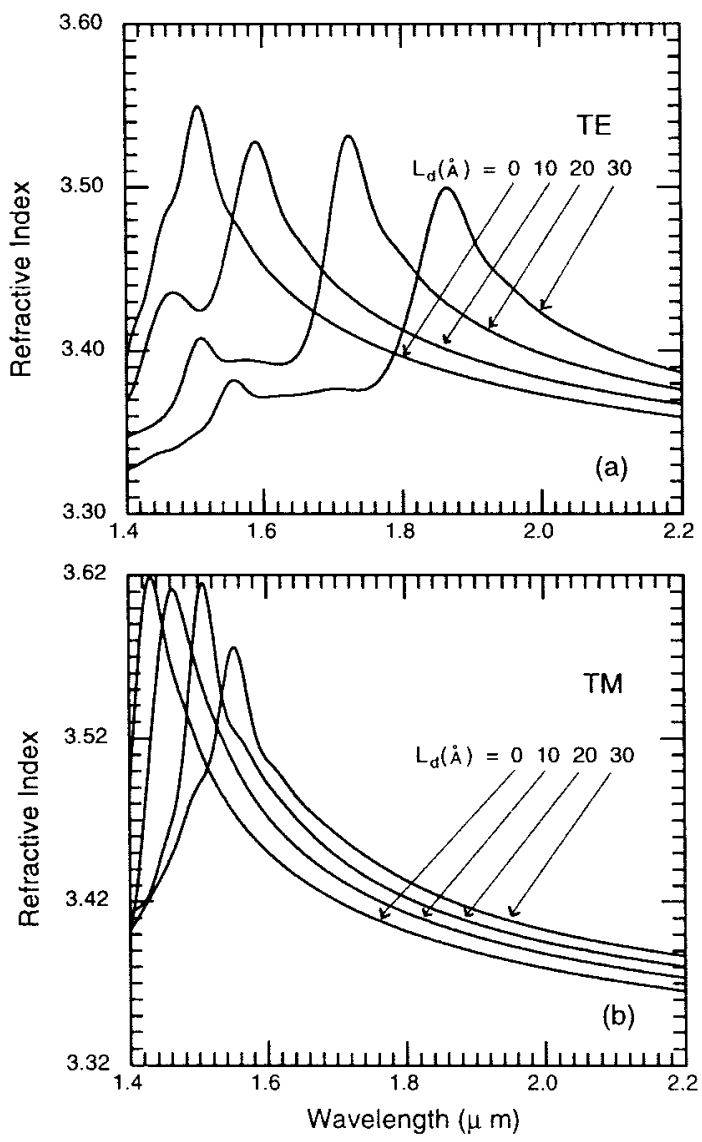

Fig. 6. The refractive index spectra of the $\mathrm{In}_{0.53} \mathrm{Ga}_{0.47} \mathrm{As} / \mathrm{InP}$ Group III interdiffused QW with $L d=0,10,20$, and $30 \AA$. (a) TE polarization. (b) TM polarization.

depth, as is reflected in its absorption spectrum. There is only one light hole peak for the TM refractive index spectra.

The refractive index spectra for the TE and TM polarizations (Group V interdiffusion) in the wavelength range 1.1-2.4 $\mu \mathrm{m}$ is shown in Fig. 7(a) and (b), respectively. The general features of the spectra is similar to the case of Group III interdiffusion. The main difference is that interdiffusion produces a blueshifted spectra to the shorter wavelengths.

Comparable interdiffusion rates on the two sublattices are possible and it has been observed in intermixed InGaAs-InP QW's induced by sulphur diffusion [25], silicon diffusion [26], phosphorus-ion implantation [27], and impurity-free intermixing through repetitive thermal annealing [28]. The effect of both sublattices intermixing on the optical properties of this material system is also of importance. The different rates of interdiffusion are characterized by relating the two diffusion lengths through a ratio defined as $r=L_{d}^{\mathrm{V}} / L_{d}^{\mathrm{III}}$. When $r<1$, the interdiffusion rate of the Group III sublattice is larger than that of the Group V sublattice, and vice versa for $r>1$. For $r=1$, an identical rate prevails and the latticematched condition is maintained. Fig. 8 shows the TE and TM refractive index spectra for $r=1, r=0.5$ (double Group-III rate) and $r=2$ (double Group-V rate). The $L_{d}^{\mathrm{III}}$ is fixed at $30 \AA$, for lattice-matched as-grown $L_{z}=60 \AA$. These spectra demonstrate a blue and red shifting from the $r=1$ refractive index, which represent a more dominate Group-V and Group- 

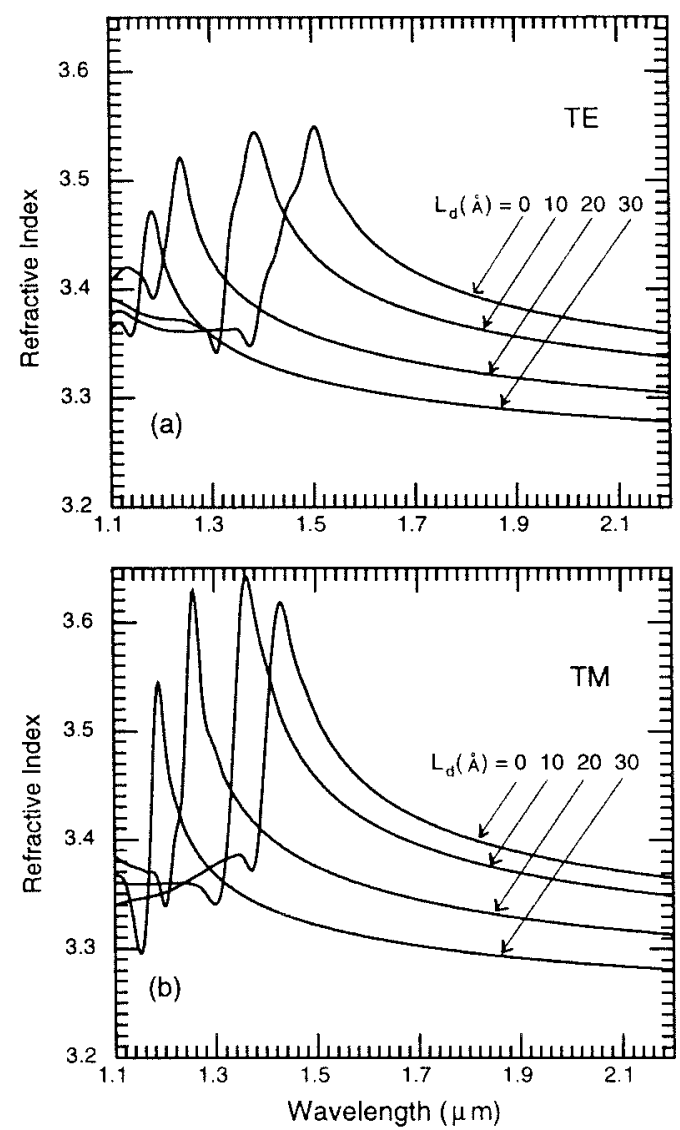

Fig. 7. The refractive index spectra of $\operatorname{In}_{0.53} \mathrm{Ga}_{0.47} \mathrm{As}-\mathrm{InP}$ Group $\mathrm{V}$ interdiffused QW with $L_{d}=0,10,20$, and $30 \AA$. (a) TE polarization. (b) TM polarization.

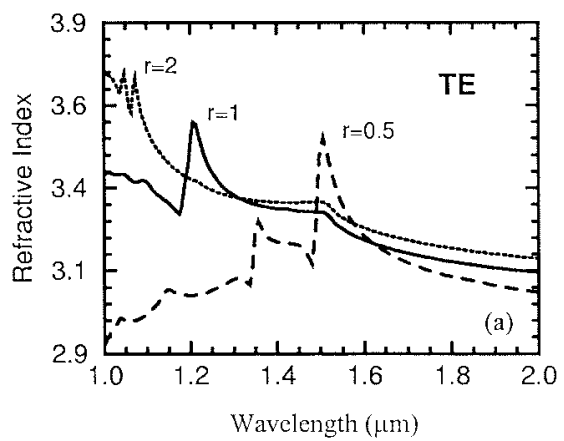

(a)

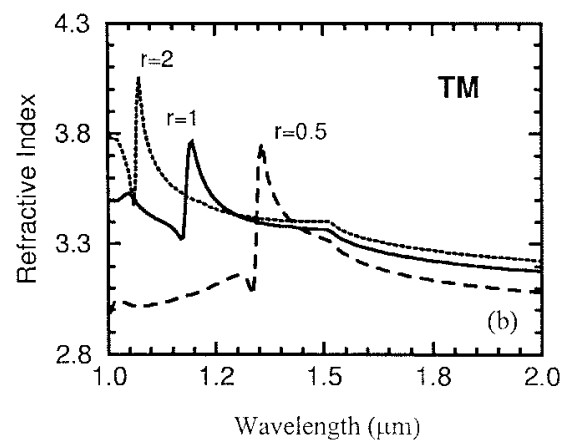

(b)

Fig. 8. The refractive index spectra of $\mathrm{In}_{0.53} \mathrm{Ga}_{0.47} \mathrm{As}-\mathrm{InP}$ Groups III and $\mathrm{V}$ interdiffused $\mathrm{QW}, r=L_{d}^{\mathrm{V}} / L_{d}^{\mathrm{III}}$ denotes their diffusion ratio with $L_{d}^{\mathrm{III}}$ fixed at $30 \AA$ and $L_{z}=60 \AA$. (a) TE polarization. (b) TM polarization.
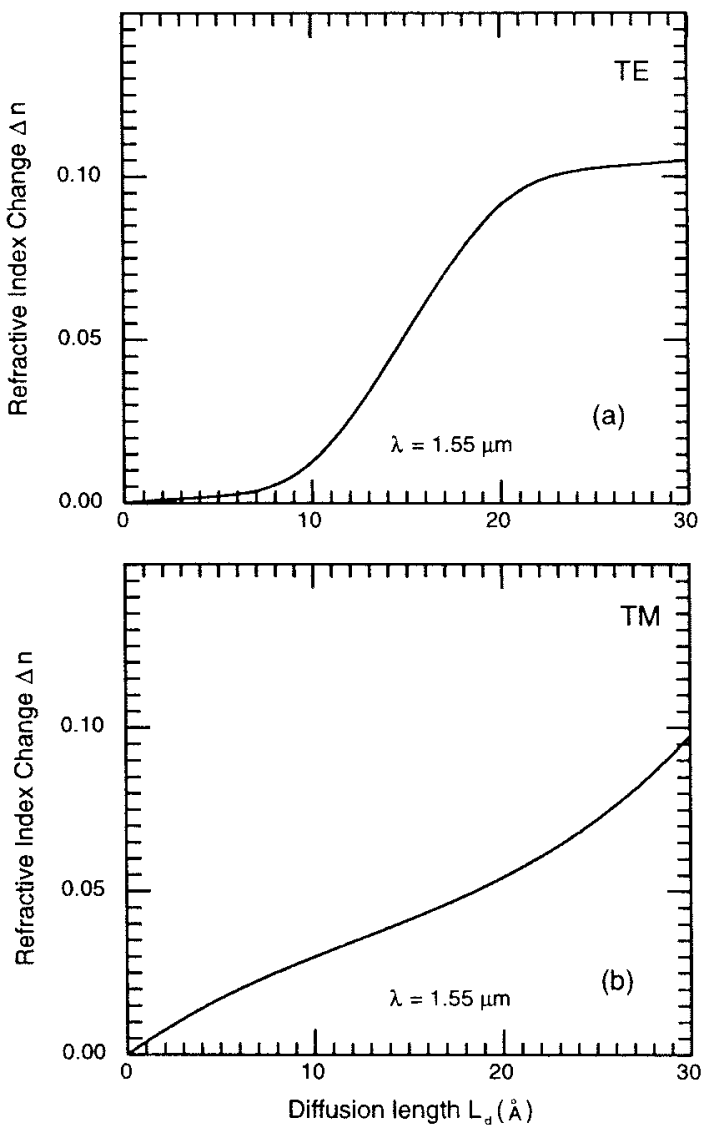

Fig. 9. Absolute refractive index difference between as-grown square QW and Group III interdiffused QW, $n\left(L_{d}>0\right)-n\left(L_{d}=0\right)$, as a function of diffusion length. (a) TE polarization. (b) TM polarization.

III interdiffusion, respectively. This phenomenon is the result of an introduction of the compressive and tensile strains in the QW for the respective interdiffusions as effectively observed in the cases of Group-III-only and Group-V-only interdiffusion. There is, however, a difference at the tail (longer wavelength end) of the spectra. The red shift produces a lower refractive index while the blue shift results in a higher one. This is in part due to the fact that both sublattices are interdiffusing which results in a more complicated behavior of the spectra movement or refractive index change. The interesting application is that at these longer wavelengths one can adjust a higher or lower refractive index by only controlling the rate of the Group-V sublattice, since the $L_{d}^{\mathrm{III}}$ is fixed in these cases.

The absolute change of refractive index for Group III interdiffusion is shown in Fig. 9. The wavelength of interest is chosen to be $1.55 \mu \mathrm{m}$ because of device application interests. From the TE case shown in Fig. 9(a), we can observe that there is an abrupt change in the refractive index when the diffusion length increases from 10 to $20 \AA$. It is because the peak of the refractive index dispersion at these diffusion lengths has crossed over the $1.55-\mu \mathrm{m}$ wavelength. For the TM case, the change is monotonic with increasing diffusion length. The absolute change of refractive index for Group V interdiffusion is shown in Fig. 10 at the same wavelength range of interest. Both cases show monotonic index change with increasing interdiffusion. 

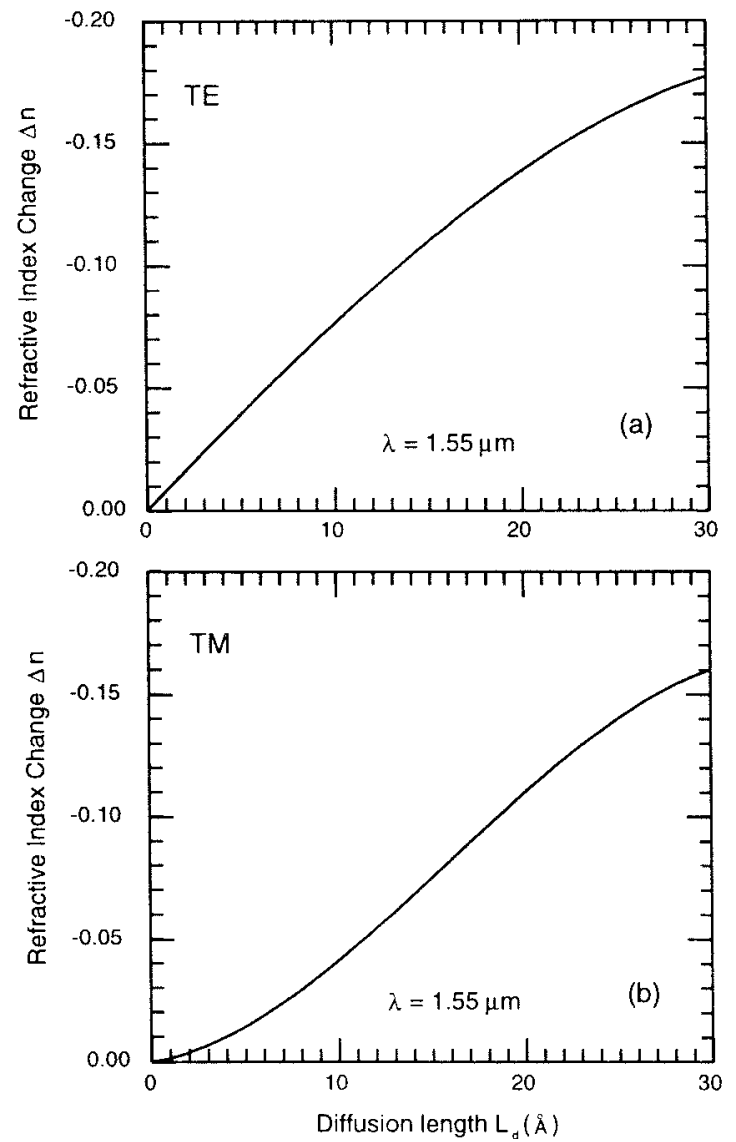

Fig. 10. Absolute refractive index difference between as-grown square $\mathrm{QW}$ and Group V interdiffused QW, $n\left(L_{d} \geq 0\right)-n\left(L_{d}=0\right)$, as a function of diffusion length. (a) TE polarization. (b) TM polarization.

The birefringence of the $\mathrm{In}_{0.53} \mathrm{Ga}_{0.47} \mathrm{As}-\mathrm{InP} \mathrm{QW}$ has also been analyzed under Group V interdiffusion (see Fig. 11). The wavelengths of interest are optimized to fall within 1.52-1.57 $\mu \mathrm{m}$. The birefringence of the as-grown square QW varies (from 0.027 to 0.007 ) over this range of wavelengths by 0.02 . However, for the interdiffused QW's, the birefringence is nearly constant over the entire wavelength range under investigation. As diffusion length increases, the birefringence approaches nearly zero. A result of less than 0.005 birefringence is obtained for the diffusion length of $30 \AA$. This will have important applications for polarization-insensitive devices [29]. Although increasing the diffusion length may lead to even less birefringence, lattice mismatch due to the tensile strain will result and can cause device degradation.

\section{CONCLUSION}

A comprehensive model for the index of refraction of $\mathrm{In}_{0.53} \mathrm{Ga}_{0.47} \mathrm{As}-\mathrm{InP}$ interdiffused QW structures is presented. Results of the polarized absorption coefficients and refractive index spectra in the wavelength range of 1.1-2.4 $\mu \mathrm{m}$ for both Group III and V interdiffusion have been obtained. The results obtained here are in good agreement with available measured data. These calculated results are important in a fundamental sense both theoretically and practically, especially in the regions where experimental data becomes unavailable.

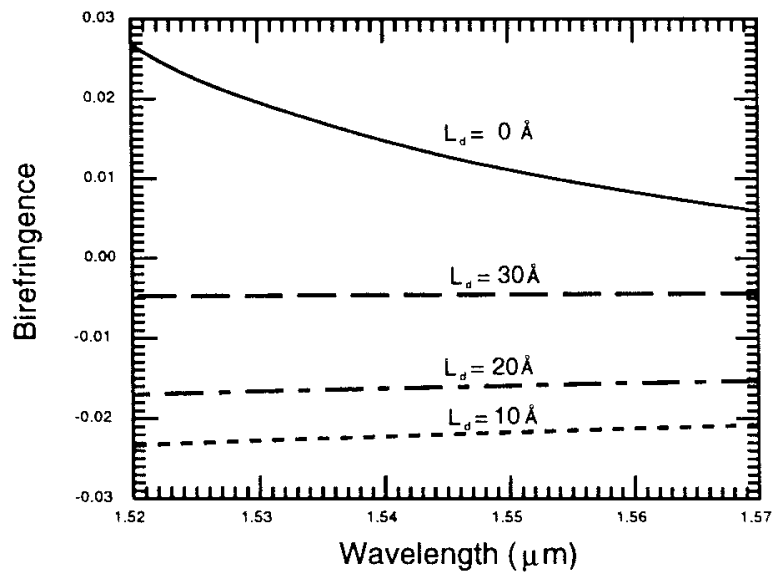

Fig. 11. Birefringence $\left(n^{\mathrm{TE}}-n^{\mathrm{TM}}\right)$ of $\mathrm{In}_{0.53} \mathrm{Ga}_{0.47} \mathrm{As}-\mathrm{InP}$ Group $\mathrm{V}$ interdiffused QW versus wavelength for $L_{d}=0,10,20$, and $30 \AA$.

The interesting part of the results shows that the refractive index spectra red shifts to longer wavelengths in the case of Group III interdiffusion and blue shifts to shorter wavelengths in the case of Group V interdiffusion. In the longer wavelengths, this produces an increase (in the former case) and a decrease (in the latter case) of the refractive index and which depend on the types of interdiffusion. The consequence is that positive and negative index steps can be engineered by controlling the interdiffusion process and is therefore attractive from the point of view of device applications. In much the same manner, the red and blue shift of the absorption edge can be used to realize low loss and mode filtering devices in addition to a more versatile wavelength tuning application. For the small birefringence properties of the Group $\mathrm{V}$ interdiffusion, there is no doubt it will find its usage in polarization-insensitive modulation devices with low loss and operated in a range of tunable operation wavelengths around $1.55 \mu \mathrm{m}$.

\section{REFERENCES}

[1] See, for instance, E. H. Li, Ed., Quantum Well Mixing and Optoelectronic Device Applications, Milestone Series. Bellingham, WA: SPIE Press, 1998.

[2] S. S. Rao, W. P. Gillin, and K. P. Homewood, "Interdiffusion of the group-III sublattice in In-Ga-As-P/In-Ga-As-P and In-Ga-As heterosturctures," Phys. Rev. B, vol. 50, pp. 8071-8073, 1994.

[3] C. Francis, F. H. Julien, J. Y. Emery, R. Simes, and L. Goldstein, "Selective band-gap blueshifting of InGaAsP/InGaAs(P) quantum-wells by thermal intermixing with phosphorus pressures and dielectric copping," J. Appl. Phys., vol. 75, pp. 3607-3610, 1994.

[4] J. Oshinowo, A. Forchel, D. Gruzmacher, M. Stollenwerk, M. Heuken, and K. Heime, "Photoluminescence study of interdiffusion in $\mathrm{In}_{0.53} \mathrm{Ga}_{0.47} \mathrm{As} / \mathrm{InP}$ surface quantum wells," Appl. Phys. Lett., vol. 60, pp. 2660-2662, 1992.

[5] H. Peyre, F. Alsina, J. Camassel, J. Pascual, and R. W. Glew, "Thermal stability of InGaAs/InGaAsP quantum wells," J. Appl. Phys., vol. 73, pp. 3760-3768, 1993.

[6] S. Sudo, H. Onishi, Y. Nakano, Y. Shimogaki, K. Tada, M. J. Mondry, and L. A. Coldren, "Impurity-free disordering of InGaAs/InGaAlAs quantum wells on InP by dielectric thin cap films and characterization of its In-plane spatial resolution," Jpn. J. Appl. Phys., vol. 35, pp. 1276-1279, 1996.

[7] J. Z. Wan, D. A. Thompson, and J. G. Simmons, "Ion implantation induced compositional intermixing in the InGaAs/InP MQW system for wavelength shifted waveguides," Nucl. Instrum. Methods B, vol. 106, pp. 461-465, 1995. 
[8] S. A. Pappert, W. Xia, X. S. Jiang, Z. F. Guan, B. Zhu, Q. Z Liu, L. S. Yu, A. R. Clawson, P. K. L. Yu, and S. S. Lau, "Planar 1.3 and $1.55 \mu \mathrm{m} \mathrm{InGaAs}(\mathrm{P}) / \mathrm{InP}$ electroabsorption waveguide modulators using oxygen ion mixing and the photoelastic effect," J. Appl. Phys., vol. 75, pp. 4352-4361, 1994.

[9] J. P. Noel, D. Melville, T. Jones, F. R. Sheperd, C. J. Miner, N. Puetz, K. Fox, P. J. Poole, Y. Feng, E. S. Koteles, S. Charbonneau, R. D. Goldberg, and I. V. Mitchell "High-realiability blue-shifted InGaAsP/InP lasers" Appl. Phys. Lett., vol. 69, pp. 3516-3518, 1996.

[10] E. S. Koteles, S. Charbonneau, P. Poole, J. J. He, M. Davies, M. Dion, G. Aers, Y. Feng, I. V. Mitchell, and R.D. Goldberg, "Photonic integration using quantum well shape modification," Phys. Canada, Sept./Oct. 1996, pp. $251-255$.

[11] G. M. Alman, L. A. Molter, H. Shen, and M. Dutta, "Refractive index approximations from linear perturbation theory for planar MQW waveguides," IEEE J. Quantum Electron., vol. 28, pp. 650-657, 1992.

[12] Y. Suzuki, H. Iwamura, T. Miyazawa, A. Wakatsuki, and O. Mikami, "Polarization-dependent refractive-index change induced by superlattice disordering," IEEE J. Quantum Electron., vol. 32, pp. 1922-1931, 1996.

[13] A. Wakatsuk, H. Iqamura, Y. Suzuki, T. Miyazawa, and O. Mikami, "Refractive index change of GaInAs/InP disordered superlattice waveguide," IEEE Photon. Technol. Lett., vol. 3, pp. 905-907, 1991.

[14] J. I. Pankove, Optical Processes in Semiconductors. New York: Dover, 1971, ch. 4.

[15] F. Bassani and G. P. Parravicini, Electronic States and Optical Transitions in Solids. Oxford, U.K.: Pergamon, 1975, p. 154.

[16] W. C. Shiu, J. Micallef, I. Ng, and E. H. Li, "Effects of different cation and anion interdiffusion rates in disordered $\mathrm{InGaAs} / \mathrm{InP}$ single quantum wells," Jpn. J. Appl. Phys., vol. 34, pp. 1778-1783, 1995.

[17] E. H. Li, K. S. Chan, and B. L. Weiss, "Eigenstates and absorption spectra of interdiffused AlGaAs/GaAs multiple quantum well structures," IEEE J. Quantum Electron., vol. 32, pp. 1399-1416, 1996.

[18] S. Adachi, "Model dielectric constants of GaP, GaAs, GaSb, InP, InAs, and InSb," Phys. Rev. B, vol. 35, pp. 7454-7463, 1987.

[19] S. Adachi, "Optical properties of $\operatorname{In}_{1-x} \mathrm{Ga}_{x} \mathrm{As}_{y} \mathrm{P}_{1-y}$ alloys," Phys. Rev. B, vol. 39, pp. 12612-12621, 1989.

[20] M. C. Y. Chan and E. H. Li, "Theory of critical layer thickness of nonconstant quantum-well-width produced by interdiffusion and its optoelectronics consequence," in Symp. F: Infrared Applications of Semiconductors II, Mater. Res. Soc. 1997 Fall Meetings, Boston, MA, Dec. 1-5, 1997, paper F 9.5.

[21] D. M. Hwang, S. A. Schwartz, R. Bhat, C. Y. Chen, and T. S Ravi, "Cation diffusion in InP/InGaAs superlattices: strain built-up and relaxation," Opt. Quantum Electron., vol. 23, pp. S829-S846, 1991.

[22] J. Micallef, E. H. Li, and B. L. Weiss, "The effects of interdiffusion on the subband-edge of $\operatorname{In}_{0.53} \mathrm{Ga}_{0.47} \mathrm{As} / \mathrm{InP}$ single quantum wells," $J$. Appl. Phys., vol. 73, pp. 7524-7532, 1993.

[23] H. Temkin, S. N. G. Chu, M. B. Panish, and R. A. Logan, "Thermal stability of InGaAs/InP quantum well structures grown by gas source molecular beam epitaxy," Appl. Phys. Lett., vol. 50, pp. 956-958, 1987.

[24] J. E. Zucker, B. Tell, K. L. Jones, M. D. Divino, K. F. Brown-Goebeler, C. H. Joyner, B. I. Miller, and M. G. Young, "Large blueshifting of InGaAs/InP quantum-well band gaps by ion implantation," Appl. Phys. Lett., vol. 60, pp. 3036-3038, 1992.

[25] I. J. Pape, P. Li Kam Wa, J. P. R. David, P. A. Claxton, and P. N. Robson, "Disordering of $\mathrm{Ga}_{0.47} \mathrm{In}_{0.53} \mathrm{As} / \mathrm{InP}$ multiple quantum well layers by sulfur diffusion," Electron. Lett., vol. 24, pp. 1217-1218, 1988.
[26] M. Razeghi, O. Archer, and F. Launay, Disorder of $\mathrm{Ga}_{x} \mathrm{In}_{1-x} \mathrm{As}_{y} \mathrm{P}_{1-y}$ - InP quantum well by $\mathrm{Zn}$ diffusion," Semicond. Sci. Technol., vol. 2, pp. 793-796, 1987.

[27] B. Tell, J. Shah, P. M. Thomas, K. F. Brown-Goebeler, A. DiGiovanni, B. I. Miller, and U. Koren, "Phosphorus ion implantation induced intermixing of InGaAs-InP quantum well structures," Appl. Phys. Lett., vol. 54 no. 16, pp. 1570-1572, 1989.

[28] T. Miyazawa, H. Iwamura, O. Mikamo, and M. Naganuma, "Compositional disordering of $\operatorname{In}_{0.53} \mathrm{Ga}_{0.47}$ As InP multiquantum well structures by repetitive rapid thermal annealing," Jpn. J. Appl. Phys., vol. 28, pp. L1039-L1041, 1989.

[29] J. J. He, S. Charbonneau, P. J. Poole, G. C. Aers, Y. Feng, E. S. Koteles, R. D. Goldberg, and I. V. Mitchell, "Polarization insensitive InGaAs/InGaAsP/InP amplifiers using quantum well intermixing," Appl. Phys. Lett., vol. 69, pp. 562-564, 1996.

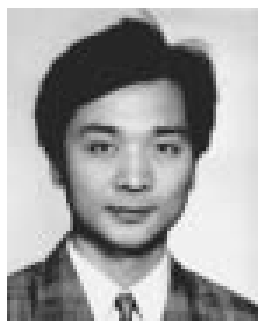

E. Herbert Li (S'87-M'88-SM'95) received the M.Phil. degree in applied mathematics and electronic engineering from the University of Hong Kong and the Ph.D. degree in electronic engineering from the University of Surrey, Surrey, U.K.

He worked for the Kirsten Aeronautical Laboratory, Seattle, WA (1979-1980). He joined KK Engineering Company, Hong Kong, as an Engineer (1981-1986) and Micro Systems, Hong Kong, as a Manager (1986-1988). He was a Member of the Faculty at the City University of Hong Kong (1988-1990). He joined the National Ion Implantation Facility at the Department of Electrical and Electronic Engineering, University of Surrey (1990-1993). He is currently a Member of the Faculty and Leader of the Optoelectronics Group, which consists of a team of 10 researchers, at the Department of Electrical and Electronic Engineering, University of Hong Kong (1994-present). He is a Visiting Scholar at Harvard University, Cambridge, MA, a Guest Professor at the Institute of Semiconductors, Shandong University, China, and a Visiting Professor at the University of Waterloo, Canada. His current research interests are mainly concerned with optoelectronic device fabrication, modeling, and characterization, and in particular interdiffusion-induced modification of quantum-well structures for the advanced performance and integration of optoelectronic devices. Since 1990, he has published over 120 international technical papers, two book chapters, and three books in the above areas. He served on both the Technical Program and International Advisory Committees of OECC in 1996 (Japan) and 1997 (Korea). He is an Editor of the International Journal of Optoelectronics.

Prof. $\mathrm{Li}$ is a "Distinguished Lecturer" of the IEEE Electron Device Society (EDS) since 1997, and he is an AdCom ex officio member and serves on several EDS committees. He received the J. Langham Thompson Premium Prize in 1992 from the Institution of Electrical Engineers (U.K.) and the Distinguished Pioneering Projects Award in 1989 from HKCSS (Hong Kong). His biography is published in Who's Who in Science and Engineering. 